\title{
RELACIÓN ENTRE LOS APOYOS INSTRUMENTAL Y EMOCIONAL RECIBIDOS Y LAS CARACTERÍSTICAS SOCIODEMOGRÁFICAS DE LOS PARTICIPANTES EN UNA MUESTRA DE POBLACION ESPAÑOLA
}

\author{
Alejandro Canedo García \\ Universidad de León \\ acang@unileon.es
}

Recepción Artículo: 27 octubre 2021 Admisión Evaluación: 27 octubre 2021 Informe Evaluador 1: 28 octubre 2021

Informe Evaluador 2: 29 octubre 2021 Aprobación Publicación: 30 octubre 2021

\section{RESUMEN}

Objetivos: Examinar la prevalencia de los apoyos instrumental y emocional recibidos y estudiar diversas características sociodemográficas en una población española de diferentes edades y grupos sociales. Métodos: Utilizamos el instrumento Acción Conjunta Intergeneracional (ACIG), un cuestionario de 88 ítems. La encuesta online fue cumplimentada por 2013 participantes (608 hombres y 1405 mujeres) que tenían una edad media de 33,96 ( $D E=16,01)$ años. Resultados: El apoyo instrumental y el apoyo emocional fueron significativamente menos prevalentes entre los participantes mayores, hombres, personas con bajo nivel educativo, bajo nivel de autonomía, jubilados y con bajo nivel de ingresos, sugiriendo pobres interacciones sociales en estos grupos poblacionales.

Palabras clave: apoyo; emociones; interacciones sociales

\section{ABSTRACT}

Relationship between instrumental and emotional support received and sociodemographic characteristics of participants in a spanish population sample. Objectives: To examine the prevalence of instrumental and emotional support received and to study various sociodemographic characteristics in a Spanish population of different ages and social groups. Methods: We used the Joint Intergenerational Action instrument (ACIG), an 88-item questionnaire. The online survey was completed by 2013 participants (608 men and 1405 women) who had a mean age of 33.96 (SD = 16.01) years. Results: Instrumental support and emotional support were significantly less prevalent among older participants, men, people with a low educational level, a low level of autonomy, retirees and with a low level of income, suggesting poor social interactions in these population groups.

Keywords: support; emotions; social interactions 


\section{RELACIÓN ENTRE LOS APOYOS INSTRUMENTAL Y EMOCIONAL RECIBIDOS Y LAS CARACTERÍSTICAS SOCIODEMOGRÁFICAS DE LOS PARTICIPANTES EN UNA MUESTRA DE POBLACIÓN ESPAÑOLA}

\section{INTRODUCCIÓN}

En las últimas décadas, ha habido un aumento notable en el número de personas mayores en todo el mundo. Actualmente, los adultos de 65 años o más representan el 8,5\% de la población mundial y se estima que este número se duplicará de forma que los adultos mayores comprenderán el 16,7\% de la población para el 2050, resultando en 1,6 mil millones de adultos mayores en todo el mundo (Organización Mundial de la Salud, 2020). Este hecho, unido al creciente aislamiento social de los adultos mayores, está llevando a los países desarrollados a promover los lazos sociales y las relaciones interpersonales con el fin de lograr un envejecimiento activo y lo que este concepto conlleva, incluyendo la promoción del bienestar, la calidad de vida y la participación activa en la sociedad de las personas mayores (Wilson \& Saklofske, 2018).

Una posible forma de construir relaciones interpersonales es desarrollar un mayor capital social dentro de las comunidades locales. El capital social se define como las redes sociales que comparten normas, valores y conocimientos, facilitando la cooperación dentro de un grupo o de diferentes grupos de población (Grootaert \& van Bastelaer, 2002). Distintos estudios han demostrado que el capital social tiene un efecto positivo en varios resultados de salud, incluidos la mortalidad, la hospitalización, la salud autopercibida y la depresión (De Silva et al., 2005; Kondo et al., 2009; Gilbert et al., 2013).

Sin embargo, todavía no hay suficiente evidencia sobre los aspectos sociales de las percepciones y estereotipos relacionados con la edad y no está del todo claro si las experiencias sociales y la participación social de diferentes grupos de edad afectan sus creencias sobre el envejecimiento. Por tanto, el objetivo de este estudio fue examinar la prevalencia del apoyo instrumental y emocional recibidos, en una muestra de población española de diferentes edades y perteneciente a diferentes grupos sociales.

\section{OBJETIVOS DE LA INVESTIGACIÓN}

Examinar si la recepción de apoyo instrumental y emocional se asociaba con las variables sociodemográficas de los encuestados.

\section{MUESTRA Y/O PARTICIPANTES}

Los participantes en este estudio fueron sujetos residentes en España de cualquier edad y de diferentes grupos sociales que respondieron un cuestionario electrónico. Un total de 2013 personas (608 hombres y 1405 mujeres) completaron la encuesta y se incluyeron en el estudio final.

\section{METODOLOGÍA Y/O INSTRUMENTOS UTILIZADOS}

\section{Instrumento}

Este estudio empleó el instrumento Acción Conjunta Intergeneracional (ACIG), previamente validado (Canedo-García et al., 2019). Consiste en un cuestionario online que, a través de 6 escalas y 14 subescalas, analiza la información aportad por personas de todas las edades en relación al apoyo instrumental y emocional que reciben y una serie de variables psicosociales.

El cuestionario también recogió datos sociodemográficos de los participantes.

\section{Procedimiento}

Los participantes fueron reclutados y completaron el cuestionario online a través del sitio web de encuestas profesionales Survey Monkey (España) durante octubre de 2017.

\section{Análisis de los datos}

Se realizó un análisis descriptivo de los datos utilizando SPSS versión \# (IBM, Armonk, NY, Estados Unidos). Las asociaciones entre variables cualitativas se evaluaron mediante un análisis de tablas de contingencia de chicuadrado. 


\section{RESULTADOS ALCANZADOS}

Con respecto a su edad, se encontró una asociación muy significativa con el apoyo tanto instrumental como emocional de los participantes. Los participantes de mayor edad ( $\geq 40$ años) fueron el grupo de personas que recibieron estos apoyos con menor frecuencia. El género de los encuestados también se asoció fuertemente con el apoyo emocional, pero solo tendió a ser significativo para el apoyo instrumental. Así, las mujeres recibían apoyo instrumental y emocional con más frecuencia que los hombres.

En relación al nivel educativo, los participantes con estudios universitarios recibían apoyo instrumental y emocional con mayor frecuencia que aquellos con bajo nivel educativo. Esta asociación fue significativa para el apoyo emocional, pero no para el apoyo instrumental recibido por los participantes. También encontramos asociaciones significativas en relación con la situación laboral y el nivel de ingresos de los participantes. El apoyo emocional, pero no el instrumental, recibido fue significativamente menos frecuente entre los participantes jubilados y el apoyo tanto instrumental como emocional entre los participantes de bajos ingresos. Sorprendentemente, en términos de su nivel de autonomía, Ios participantes que necesitaban apoyo familiar, profesional o de otro tipo recibían apoyo instrumental y emocional con mucha menos frecuencia que aquellos que no necesitaban ningún apoyo. 
Tabla 1. Asociación entre el apoyo instrumental y emocional recibido y las características sociodemográficas

\begin{tabular}{|c|c|c|c|c|c|c|c|c|}
\hline \multirow{2}{*}{ Variables } & \multicolumn{4}{|c|}{ Apoyo instrumental } & \multicolumn{4}{|c|}{ Apoyo emocional } \\
\hline & No & Si & $\chi^{2}$ & $p$ & No & Si & $\chi^{2}$ & $p$ \\
\hline \multicolumn{9}{|l|}{ Edad (años) } \\
\hline$<22$ & $\begin{array}{c}451 \\
(25.6)\end{array}$ & $\begin{array}{c}40 \\
(46.5)\end{array}$ & & & $\begin{array}{c}228 \\
(22.5)\end{array}$ & $\begin{array}{c}252 \\
(31.1)\end{array}$ & & \\
\hline $22-39$ & $\begin{array}{c}678 \\
(38.5)\end{array}$ & $\begin{array}{c}30 \\
(34.9)\end{array}$ & 20.691 & $<.000$ & $\begin{array}{c}366 \\
(36.2)\end{array}$ & $\begin{array}{c}334 \\
(41.2)\end{array}$ & 38.904 & $<.000$ \\
\hline$\geq 40$ & $\begin{array}{c}631 \\
(35.9) \\
\end{array}$ & $\begin{array}{c}16 \\
(18.6) \\
\end{array}$ & & & $\begin{array}{c}418 \\
(41.3) \\
\end{array}$ & $\begin{array}{c}225 \\
(27.7) \\
\end{array}$ & & \\
\hline \multicolumn{9}{|l|}{ Género } \\
\hline $\begin{array}{l}\text { Hombre } \\
\text { Mujer }\end{array}$ & $\begin{array}{c}548 \\
(31.1) \\
1216 \\
(68.9) \\
\end{array}$ & $\begin{array}{c}19 \\
(22.1) \\
67 \\
(77.9) \\
\end{array}$ & 3.106 & .078 & $\begin{array}{c}349 \\
(34.4) \\
667 \\
(65.6) \\
\end{array}$ & $\begin{array}{c}217 \\
(26.8) \\
594 \\
(73.2) \\
\end{array}$ & 12.161 & $<.000$ \\
\hline \multicolumn{9}{|l|}{ Lugar de origen } \\
\hline \multicolumn{9}{|l|}{ Educación } \\
\hline $\begin{array}{r}\text { Estudios inferiores a universitarios } \\
\text { Estudios universitarios }\end{array}$ & $\begin{array}{c}305 \\
(17.3) \\
1459 \\
(82.7) \\
\end{array}$ & $\begin{array}{c}23 \\
(26.7) \\
63 \\
(73.3) \\
\end{array}$ & 5.025 & .025 & $\begin{array}{c}182 \\
(17.9) \\
834 \\
(17.6) \\
\end{array}$ & $\begin{array}{c}143 \\
(82.1) \\
668 \\
(82.4) \\
\end{array}$ & .024 & .876 \\
\hline \multicolumn{9}{|l|}{ Nivel de autonomía } \\
\hline Apoyo familiar/profesional/otro & $\begin{array}{c}1528 \\
(86.6) \\
236 \\
(13.4) \\
\end{array}$ & $\begin{array}{c}57 \\
(66.3) \\
29 \\
(33.7) \\
\end{array}$ & 27.650 & $<.000$ & $\begin{array}{c}904 \\
(89.0) \\
112 \\
(11.0) \\
\end{array}$ & $\begin{array}{c}661 \\
(81.5) \\
150 \\
(18.5) \\
\end{array}$ & 20.498 & $<.000$ \\
\hline \multicolumn{9}{|l|}{ Estado civil } \\
\hline \multicolumn{9}{|l|}{ Forma de convivencia } \\
\hline $\begin{array}{r}\text { Vive solo/con hijos/con nietos } \\
\text { Vive con pareja/con pareja e hijos/o } \\
\text { nietos } \\
\text { Vive con padres y/o abuelos/con otros } \\
\text { familiares } \\
\text { Vive con amigos/otros tipos }\end{array}$ & $\begin{array}{c}220 \\
(12.5) \\
644 \\
(36.5) \\
546 \\
(31.0) \\
354 \\
(20.1) \\
\end{array}$ & $\begin{array}{c}10 \\
(11.6) \\
16 \\
(18.6) \\
37 \\
(43.0) \\
23 \\
(26.7) \\
\end{array}$ & 12.999 & .005 & $\begin{array}{c}183 \\
(13.6) \\
395 \\
(38.9) \\
310 \\
(30.5) \\
173 \\
(17.0) \\
\end{array}$ & $\begin{array}{c}91 \\
(11.2) \\
261 \\
(32.2) \\
260 \\
(32.1) \\
199 \\
(24.5) \\
\end{array}$ & 20.477 & $<.000$ \\
\hline \multicolumn{9}{|l|}{ Situación laboral } \\
\hline $\begin{array}{l}\text { Desempleado } \\
\text { Empleado } \\
\text { Jubilado }\end{array}$ & $\begin{array}{c}778 \\
(44.1) \\
846 \\
(48.0) \\
140(7.9)\end{array}$ & $\begin{array}{c}48 \\
(55.8) \\
31 \\
(36.0) \\
7(8.1)\end{array}$ & 4.977 & .083 & $\begin{array}{c}400 \\
(39.9) \\
513 \\
(50.5) \\
103 \\
(10.1) \\
\end{array}$ & $\begin{array}{c}414 \\
(51.0) \\
354 \\
(43.6) \\
43(5.3)\end{array}$ & 31.477 & $<.000$ \\
\hline \multicolumn{9}{|l|}{ Nivel de ingresos (€/mes) } \\
\hline $\begin{array}{r}>2001 \\
1001-2000 \\
<1000\end{array}$ & $\begin{array}{c}985 \\
(55.8) \\
470 \\
(26.6) \\
309 \\
(17.5) \\
\end{array}$ & $\begin{array}{c}64 \\
(74.4) \\
15 \\
(17.4) \\
7(8.1)\end{array}$ & 11.863 & .003 & $\begin{array}{c}521 \\
(51.3) \\
276 \\
(27.2) \\
219 \\
(21.6) \\
\end{array}$ & $\begin{array}{c}512 \\
(63.1) \\
204 \\
(25.2) \\
95 \\
(11.7) \\
\end{array}$ & 37.314 & $<.000$ \\
\hline
\end{tabular}

NOTA: $\mathrm{N}=$ número de participantes, $\chi^{2}=$ test Chi-cuadrado, $p=$ significación

Riesgo $\alpha=.05$

Fuente: elaboración propia 


\section{DISCUSIÓN}

Los adultos mayores suelen recibir apoyo social de familiares o de profesionales de la salud o de servicios sociales, pero también pueden ofrecer diversos tipos de apoyo a otras personas (Fernández Ballesteros et al., 2011).

En nuestro estudio, los apoyos instrumental y emocional fueron significativamente menos prevalentes entre Ios participantes de mayor edad, los hombres, las personas con bajo nivel educativo, con bajo nivel de autonomía, jubilados y con un nivel de ingresos bajo, lo que sugiere que estos grupos de población tienen escaso compromiso social y juegan roles menos productivos en la comunidad.

\section{CONCLUSIONES}

Nuestro estudio proporciona información sobre la prevalencia de recibir apoyo instrumental y emocional en un grupo de población española y cómo influye en el compromiso social de diferentes grupos de edad y sociales. Por lo tanto, la identificación de las características socioculturales de los adultos con escasa participación en la sociedad es fundamental para que los gobiernos e instituciones desarrollen estrategias útiles para promover la participación social y los beneficios asociados para los adultos mayores y personas de otras edades.

\section{REFERENCIAS BIBLIOGRÁFICAS}

Canedo-García, A., García-Sánchez J. N. \& Pacheco-Sanz, D. I. (2019). Acción conjunta intergeneracional (acig). Descripción de variables intervinientes. International Journal of Developmental and Educational Psychology. Revista INFAD de Psicología, 3(1), 143-148. https://doi.org/10.17060/ijodaep.2019.n1.v3.1458

De Silva, M. J., McKenzie, K., Harpham, T. \& Huttly, S. R. (2005). Social capital and mental illness: a systematic review. Journal of Epidemiology and Community Health, 59(8), 619-627. https://doi.org/10.1136/jech.2004.029678.

Gilbert, K. L., Quinn, S. C., Goodman, R. M., Butler, J. \& Wallace J. (2013). A meta-analysis of social capital and health: a case for needed research. Journal of Health Psychology, 18(11), 1385-1399. https://doi.org/10.1177/1359105311435983.

Grootaert, C. \& van Bastelaer, T. (2002). Understanding and Measuring Social Capital: A Multidisciplinary Tool for Practitioners. Directions in Development. Washington, DC: World Bank.

Kondo, N., Sembajwe, G., Kawachi, I., van Dam, R. M., Subramanian, S. V. \& Yamagata, Z. (2009). Income inequality, mortality, and self-rated health: meta-analysis of multilevel studies. BMJ, 339, b4471. https://doi.org/10.1136/bmj.b4471.

Wilson, C. A. \& Saklofske, D. H. (2018). The relationship between trait emotional intelligence, resiliency, and mental health in older adults: the mediating role of savouring. Aging \& Mental Health, 22(5), 646-654. http://doi.org/10.1080/13607863.2017.1292207

World Health Organization. (2020). Decade of healthy ageing: Baseline report. WHO. 
